Atlas du Québec - L'agriculture. Bureau de recherches économiques, Ministère de l'Industrie et du commerce, 1966. 47 cartes.

LÉTOURNEAU, Firmin, La Côte nord de Gaspé - Les premières tentatives d'établissement, les vrais fondateurs, les paroisses actuelles et un programme de développement. — Revue d'Histoire de la Gaspésie, vol. III, no IV, octobre-décembre 1965 : 176-218.

Culture, vol. XXVII, no 3 (septembre 1966) Des pages 230 à 349, « En hommage aux R.P. Ephrem Longpré, o.f.m., savant théologien médiéviste canadien".

Répertoire des Ministères canadiens depuis la Confédération — Supplément 1er janvier 1957 - 1er août 1965. Archives publiques du Canada, Ottawa, 1966. $21 \mathrm{p}$.

Recent Acquisitions - National Portrait Gallery Smithsonian Institution. Entries Compiled by Robert G. Stewart. Smithsonian Institution, Washington, D.C. 1966.

Archives publiques du Canada - Division des manuscrits - Inventaire provisoire. Fonds des manuscrits No 18. Documents antérieurs à la cession. Ottawa, 1966.

Noël à Ville-Marie en 1665 et Messe de Minuit à l'Hôtel-Dieu il y a 300 ans - Christmas at Ville-Marie in 1665 Midnight Mass at Hôtel-Dieu, Montreal Three Hundred Years. Texte : Louis-Raoul de Lorimier - Illustrations: James Mclsaac, 23 p.

La Louisiane française - Exposition de documents du 25 mai au 19 juin 1966. Musée du Québec.

LA FERRIÈRE, Philippe, Les origines de la Bibliothèque Saint-Sulpice et ses trésors - Conférence faite à la Bibliothèque Saint-Sulpice le 27 avril 1966.

Répertoire des thèses en cours portant sur des sujets d'histoire et autres sujets connexes compilés par les Archives publiques du Canada et édités par la Société historique du Canada - no 1, 1966. $39 \mathrm{p}$.

TESIOROWSKI, Jan, Canadiens français - Puissance du nombre. Etude statistique sur le bilinguisme. Présentation Lt.-CoI. Sarto Marchand,

président Conseil d'Expansion Economique Inc., et de la Fondation Melchers Limitée. Beauchemin, mars $1966.50 \mathrm{p}$

Toponymie - Bulletin d'information no 4 (juin 1966). Service d'Urbanisme, Ville de Montréal. $149 \mathrm{p}$

MARION, Séraphin, de la Société royale du Canada et des " Dix ", Péché d'omission d'un historien canadien. Ottawa, juin 1966. Brochure de 20 p.

Cahiers d'Archéologie Québécoise, mai 1966 - 2e année, no 1, Station du Sylvicole moyen à la-Pointe-du-Lac - Matériel archaïque de Bécancour. Le Musée d'Archéologie - Centre des études universitaires, Trois-Rivières. \$1. l'ex. 41 p.

ROMME, Jules, O.Prém., Lacolle, 1865-1965.

Volume 20, numéro 3, décembre 1966

URI : https://id.erudit.org/iderudit/302610ar

DOI : https://doi.org/10.7202/302610ar

Aller au sommaire du numéro

Éditeur(s)

Institut d'histoire de l'Amérique française

ISSN

0035-2357 (imprimé)

1492-1383 (numérique)

Découvrir la revue

Citer ce compte rendu

(1966). Compte rendu de [Atlas du Québec - L'agriculture. Bureau de recherches économiques, Ministère de l'Industrie et du commerce, 1966. 47 cartes. / LÉTOURNEAU, Firmin, La Côte nord de Gaspé - Les premières tentatives d'établissement, les vrais fondateurs, les paroisses actuelles et un programme de développement. - Revue d'Histoire de la Gaspésie, vol. III, no IV, octobre-décembre 1965 : 176-218. / Culture, vol. XXVII, no 3 (septembre 1966) Des pages 230 à 349, « En hommage aux R.P. Ephrem Longpré, o.f.m., savant théologien médiéviste canadien »./ Répertoire des Ministères canadiens depuis la Confédération - Supplément 1er janvier 1957 - 1er août 1965. Archives publiques du depuis la Confédération - Supplément 1er janvier 1957 - 1er août 1965. Archives publiques du
Canada, Ottawa, 1966. 21 p. / Recent Acquisitions - National Portrait Gallery Smithsonian Institution. Entries Compiled by Robert G. Stewart. Smithsonian Institution, Washington, D.C. 1966. / Archives publiques du Canada - Division des manuscrits - Inventaire provisoire. Fonds des manuscrits No 18 Documents antérieurs à la cession. Ottawa, 1966. / Noël à Ville-Marie en 1665 et Messe de Minuit à l'Hôtel-Dieu il y a 300 ans - Christmas at Ville-Marie in 1665 Midnight Mass at Hôtel-Dieu, Montreal Three Hundred Years. Texte : Louis-Raoul de Lorimier - Illustrations: James Mclsaac, 23 p. / La Louisiane fran Years. Tu FERRIĖRE, Philippe, Les origines de la Bibliothèque Saint-Sulpice et ses trésors - Conférence faite à la FERRIËRE, Philippe, Les origines de la Bibliothèque Saint-Sulpice et ses trésors - Conférence faite à
Bibliothèque Saint-Sulpice le 27 avril 1966. / Répertoire des thèses en cours portant sur des sujets Bibliothèque Saint-Sulpice le 27 avril 1966. / Répertoire des thèses en cours portant sur des sujets
d'histoire et autres sujets connexes compilés par les Archives publiques du Canada et édités par la Société historique du Canada - no 1, 1966. 39 p. / TESIOROWSKI, Jan, Canadiens français - Puissance du nombre. Etude statistique sur le bilinguisme. Présentation Lt.-CoI. Sarto Marchand, président Consei d'Expansion Economique Inc., et de la Fondation Melchers Limitée. Beauchemin, mars 1966. 50 p. Toponymie - Bulletin d'information no 4 (juin 1966). Service d'Urbanisme, Ville de Montréal. 149 p. MARION, Séraphin, de la Société royale du Canada et des " Dix ", Péché d'omission d'un historien canadien. Ottawa, juin 1966. Brochure de 20 p. / Cahiers d'Archéologie Québécoise, mai 1966 - 2e année, no 1, Station du Sylvicole moyen à la-Pointe-du-Lac - Matériel archaïque de Bécancour. Le Musée d'Archéologie - Centre des études universitaires, Trois-Rivières. \$1. l'ex. 41 p. / ROMME, Jules, O.Prém., d'Archéologie - Centre des études universitaires, Trois-Rivières. \$1.l'ex. 41

https://doi.org/10.7202/302610ar

Ce document est protégé par la loi sur le droit d'auteur. L'utilisation des services d’Érudit (y compris la reproduction) est assujettie à sa politique d'utilisation que vous pouvez consulter en ligne.

https://apropos.erudit.org/fr/usagers/politique-dutilisation/ 
Atlas du Québec - L'agriculture. Bureau de recherches économiques, Ministère de l'Industrie et du commerce, 1966. 47 cartes.

"Soucieux de présenter une image aussi complète que possible de l'activité économique du Québec, le Bureau de recherches économiques du ministère de l'Industrie et du Commerce a entrepris la publication d'une documentation cartographique portant sur les différents secteurs de l'économie québecoise.

"La présente section consacrée à l'agriculture constitue le premier chapitre d'un Atlas économique du Québec. D'autres sections se rapportant à la population, aux régions du Québec, aux industries de transformation, au secteur primaire et au secteur tertiaire, au milieu physique, etc., sont en voie de réalisation et paraîtront d'ici la fin de 1967.

"Cette première tranche n'a pas la prétention de fournir une image complète et détaillée du domaine agricole; elle se limite à une description, accompagnée de brefs commentaires, des principaux traits de l'activité agricole au Québec, envisagée sous l'aspect de sa répartition géographique.

"La dimension espace figure de plus en plus comme élément essentiel dans la connaissance du milieu économique et social et pour l'élaboration des programmes d'action du Gouvernement et de l'entreprise privée. C'est la raison d'être de l'approche cartographique que nous avons adoptée dans cette série de travaux.

"Le projet d'un Atlas économique du Québec est un travail d'envergure dont la réalisation nécessite l'aide précieuse de nombreux collaborateurs appartenant aux différents ministères du Gouvernement provincial et à l'entreprise privée."

$$
\begin{gathered}
\text { "Préface", signée GILLES MARTIN, } \\
\text { le directeur, } \\
\text { Bureau de recherches économiques. }
\end{gathered}
$$

LÉTOURnEAU, Firmin, La Côte nord de Gaspé - Les premières tentatives d'établissement, les vrais fondateurs, les parois- 
ses actuelles et un programme de développement. Présentation de M. l'abbé Claude Allard, directeur de la Revue d'Histoire de la Gaspésie et lettre-préface de M. Esdras Minville, directeur honoraire de l'Ecole des Hautes Etudes commerciales de Montréal. - Revue d'Histoire de la Gaspésie, vol. III, no IV, octobre-décembre 1965: 176-218.

Extrait de la présentation de M. l'abbé Claude Allard:

"Firmin Létourneau a fourni le texte complet et définitif [d'un numéro]: d'abord pensé en vue d'une conférence, le travail de recherche de l'auteur devient maintenant une source de références, qu'on aimera consulter à loisir et qui inspirera d'autres ouvrages sur la Côte nord de la Gaspésie.

"Il ne s'agit, en effet, que de la Côte nord, cette lisière de pays qui s'étend du Saint-Maurice-de-l'Echourie en conquête d'espace vers Rimouski, englobant au passage Murdochville, profondément enfouie à l'intérieur des terres. L'auteur a terminé son œuvre avec Saint-Norbert-de-Cap-Chat, dernière paroisse du diocèse de Gaspé et porte d'entrée vers le diocèse de Rimouski. Passé, présent, avenir surgissent et s'entremêlent dans la pensée de M. Létourneau."

Culture, vol. XXVII, no 3 (septembre 1966) Des pages 230 à 349, "En hommage aux R.P. Ephrem Longpré, o.f.m., savant théologien médiéviste canadien".

Yvonne Peter, Souvenirs personnels, 1890-1965. - Edouard Parent, Bibliographie. - Jacques-Guy Bougerol, Saint Bonaventure et la philosophie chrétienne. - David Ethelbert Flood, A Statement of P.J. Olivi on Gospel Life. - Luc Mathieu, Le fondement théologique de l'émerveillement franciscain devant le monde créé. - Richard Bergeron, Le problème de l'Antéchrist et la conversion de Newman.

Répertoire des Ministères canadiens depuis la Confédération Supplément 1er janvier 1957 - 1er août 1965. Archives publiques du Canada, Ottawa, 1966. 21 pages.

Ce répertoire a été compilé au bureau du Conseil privé par MM. J. L. Cross et A. Millar. Nous espérons qu'une nouvelle édition élaborée du répertoire couvrant la période 1867-1967, dira M. Kaye Lamb, archiviste du Dominion, soit accessible en 1967. 
Recent Acquisitions - National Portrait Gallery Smithsonian Institution. Entries Compiled by Robert G. Stewart. Smithsonian Institution, Washington, D.C. 1966.

Brochure contenant la liste de 50 portraits, récemment acquis, de gouverneurs, diplomates, etc. Reproductions de plusieurs portraits.

Archives publiques du Canada - Division des manuscrits Inventaire provisoire. Fonds des manuscrits No 18. Documents antérieurs à la cession. Ottawa, 1966.

Ce Fonds des manuscrits no 18 comprend, pour la plupart, des documents personnels et des pièces uniques qui se rattachent à la période antérieure à 1763 . Il compte un certain nombre d'originaux parmi les plus intéressants et les plus précieux que possèdent les Archives publiques du Canada, de même que des fac-similés, photostats, transcriptions et copies de microfilms de documents dont les originaux sont conservés ailleurs. Inventaire publié dans les deux langues.

Noël à Ville-Marie en 1665 et Messe de Minuit à l'Hôtel-Dieu il y a 300 ans - Christmas at Ville-Marie in 1665 Midnight Mass at Hôtel-Dieu, Montreal Three Hundred Years. Texte: Louis-Raoul de Lorimier - Illustrations: James McIsaac, 23 pages.

Cette petite brochure, gentiment illustrée, contient une lettre de Claude du Mousson, tambour au régiment de Carignan-Salières, en garnison d'hivernage à Ville-Marie, écrite à sa mère, la Marquise douairière du Mousson, à La Rochelle. Cette lettre, datée du 25e jour de décembre 1665, illustre, avec force détails, la messe de minuit à l'Hôtel-Dieu de Montréal, fait connaître ceux "des notables et colons qui étaient à la solennité, tous en leurs beaux ajustements", enfin relate par le détail le festin auquel Mousson prit part au château seigneurial.

La Louisiane française - Exposition de documents du 25 mai au 19 juin 1966. Musée du Québec.

Pour "souligner les échanges culturels qui s'élaborent entre la Louisiane et le Québec", les Archives du Québec ont présenté une importante exposition de cartes, de gravures, de documents, avec le concours de plusieurs dépôts du Canada, de France et de la Louisiane. Cette exposition portait surtout sur la Louisiane 
française, depuis le premier Etablissement du Mississipi, jusqu'en 1803, alors que le pavillon français flotte pour la dernière fois sur la Nouvelle-Orléans. Cette brochure de 16 pages contient la liste de tous les documents que le public a pu découvrir lors de cette exposition.

LA FERRIÈre, Philippe, Les origines de la Bibliothèque SaintSulpice et ses trésors - Conférence faite à la Bibliothèque Saint-Sulpice le 27 avril 1966.

Monsieur La Ferrière a voulu, par son travail, rendre un hommage tout particulier aux Messieurs de Saint-Sulpice en rappelant les origines de la bibliothèque qui porte leur nom. C'est le 12 septembre 1915 qu'a lieu l'inauguration de la Bibliothèque, sous la présidence de M. Lecocq, supérieur du Séminaire de Saint-Sulpice, Son premier conservateur en est M. Aegidius Fauteux. Une plaque de bronze, œuvre du sculpteur Alfred Laliberté, offerte par la Société historique de Montréal à la Bibliothèque Saint-Sulpice, rappelle la mémoire de ce fin lettré qui fut pendant plus de quinze ans l'âme dirigeante de la Bibliothèque. En passant, ajoutons qu'une annexe de la Bibliothèque, située rue Esplanade, à Montréal, portera désormais le nom d'Aegidius Fauteux, hommage posthume que lui rend la Province de Québec, puisque la Bibliothèque Saint-Sulpice est désormais une bibliothèque d'état.

M. La Ferrière fait la revue des trésors que contient la Bibliothèque: fonds canadien, manuscrits et archives, fonds étrangers, et nous fournit une longue nomenclature d'ouvrages anciens qui représente, aux yeux des historiens et des bibliothécaires, un grand intérêt.

Répertoire des thèses en cours portant sur des sujets d'histoire et autres sujets connexes compilés par les Archives publiques du Canada et édité par la Société historique du Canada no $1,1966.39$ pages.

Ce répertoire remplacera désormais la liste des thèses publiée annuellement jusqu'ici par la Canadian Historical Review. La liste comprendra toutes les thèses patronnées par les instituts d'histoire des universités canadiennes. De même les thèses préparées dans d'autres facultés telles que les sciences sociales, la géographie, etc., seront acceptées si elles nécessitent des recherches historiques et si elles présentent un certain intérêt pour l'historien. L'on se propose aussi d'y inclure les études sur l'his- 
toire du Canada poursuivies dans les principales universités étrangères. La Bibliothèque nationale publie annuellement une liste des thèses acceptées par les universités canadiennes. Le présent travail se limitera aux thèses en voie de préparation. Ce répertoire aura pour utilité première d'éviter que plusieurs personnes ne consacrent leur temps à la même recherche. Guide sur lequel les chercheurs pourront compter.

Tesiorowski, Jan, Canadiens français - Puissance du nombre. Etude statistique sur le bilinguisme. Présentation Lt-Col. Sarto Marchand, président Conseil d'Expansion Economique Inc., et de la Fondation Melchers Limitée. Beauchemin, mars 1966. 50 pages.

Dans la présentation de cette brochure, le lt-colonel Sarto Marchand croit opportun de porter à la connaissance du public "une étude statistique sur le bilinguisme entreprise par un néocanadien". "Il nous a paru, ajoute-t-il, que son statut de néocanadien lui conférait une possibilité plus grande d'objectivité dans l'interprétation des chiffres."

Monsieur Jan Tesiorowski est au Québec depuis treize ans. Il a pu étudier notre situation à la lumière des statistiques vitales du pays tout entier. Il présente des vues originales qui s'éloignent du pessimisme habituel de ceux qui traitent ce sujet. Exposé réconfortant, plein d'intérêt "pour tous ceux qui sont sensibles au langage des chiffres [et] pour tous ceux qui travaillent au progrès du Canada français".

Toponymie - Bulletin d'information no 4 (juin 1966). Service d'Urbanisme, Ville de Montréal. 149 pages.

M. Aimé Desautels, archiviste, dans ce Bulletin d'information, a pour but de "transmettre au lecteur les notes historiques compilées sur les rues de Montréal. Une première édition de ce répertoire a été publiée en 1961. La présente édition, basée sur la première, a été revue et corrigée, augmentée des nouveaux noms attribués depuis quatre ans et enrichie des recherches poursuivies depuis la publication antérieure.

La création du Service d'Urbanisme ne remonte qu'à 1941, sous la juridiction duquel fut placé le Sous-Comité de Toponymie actuel. Auparavant, tous les noms de rues, depuis la fondation de Montréal, étaient "consacrés par la coutume ou laissés au hasard de l'imagination des seigneurs, le plus souvent confiés à la responsabilité d'individus. 
Travail fort utile qui intéressera sûrement les amateurs de la grande et de la petite histoire.

MARION, Séraphin, de la Société royale du Canada et des "Dix", Péché d'omission d'un historien canadien. Ottawa, juin 1966. Brochure de 20 pages.

L'auteur collige ici quelques-unes des omissions qui parsèment plusieurs des ouvrages de l'historien Donald Creighton, ancien professeur d'histoire du Canada à l'Université de Toronto. Les sujets sont les suivants: L'invasion américaine de 1775 - La bataille de Châteauguay - Cartier et la Confédération - L'enrôlement des Canadiens français au cours de la première guerre mondiale.

Cahiers d'Archéologie Québecoise, mai 1966 - 2e année, no 1, Station du Sylvicole moyen à la-Pointe-du-Lac - Matériel archaïque de Bécancour. Le Musée d'Archéologie - Centre des études universitaires, Trois-Rivières. $\$ 1$. l'ex. $41 \mathrm{p}$.

L'intention du directeur, M. l'abbé Gilles Boulet, est “de mettre à la disposition de la jeune archéologie québécoise un médium d'information imprimé qui permette à chaque chercheur, à chaque groupe de transmettre aux autres ses découvertes et l'état de ses recherches. Les Cahiers seront donc d'abord un lien entre les différentes équipes qui œuvrent dans le Québec... Les Cahiers veulent aussi porter à l'extérieur la connaissance des travaux qui se font chez-nous ... Nous pourrons, grâce à eux, établir des liens, aujourd'hui inexistants, entre nos chercheurs et ceux des autres centres d'étude à travers le monde. Ces liens seront sûrement de nature à aider considérablement l'archéologie québécoise."

Une nouvelle chronique, intitulée "Noms, groupes et dates" ouvre la porte à toutes "les discussions, à tous les apports qui permettront de situer peu à peu les grandes civilisations préhistoriques du Québec dans leur contexte particulier aussi bien que dans l'ensemble nord-américain".

Romme, Jules, O.Prém., Lacolle, 1865-1965.

A l'occasion du centenaire de l'église paroissiale de SaintBernard-de-Lacolle, le 31 octobre 1965, le Père Jules Romme, O.Prém., publie une petite brochure sur l' "histoire de la Paroisse", le rôle joué par cette seigneurie dans notre Histoire. Sa 
participation à la guerre de 1814, à l'insurrection de 1837-1838 nous est relatée en quelques pages courtes et précises. La première cure est confiée à $M$. l'abbé Charles-François-Calixte Morisson, à qui succédera bientôt le curé patriote Antoine Labelle, le futur curé des "Pays d'en haut". Une description de "nos maisons centenaires" termine cette courte, mais instructive brochure.

\section{Ouvrages reçus:}

Paul-André Leclerc, L'émigration féminine vers l'Amérique française aux XVIIe et XVIIIe siècle. Tome I, Antilles. Tome II, Amérique du Nord.

André Bergevin, Cameron Nish, Anne Bourassa, Henri Bourassa, biographie. Index des écrits. Index de la correspondance publique 1895-1924.

Bona Arsenault, Histoire et généalogie des Acadiens, tome I et II.

Proceedings in Parliament 1610. The House of Commons. Edited by Elizabeth Read Foster, en 2 vol.

Dictionnaire de spiritualité, ascétique et mystique. Doctrine et histoire. Fascicule XLI.

André Beaulieu, Jean Hamelin, Les journaux du Québec de 1764 à 1964. Préface Jean-Charles Bonenfant.

S. Saint-Denis, o.s.a., Gaspésiana, 1965.

Germain Lesage, Le transfert à Nicolet des Scurs de l'Assomption de la Sainte-Vierge, 1965.

Pierre M. Conlon, Jean-François Bion et sa relation des tourments soufferts par les forçats protestants, 1966.

Cameron Nish, Le régime français 153ł-1760, vol. 1.

Adolphe Robert, Souvenirs et portraits, Manchester, New-Hampshire, 1965.

Lucien Lelièvre, L'enseignement du thomisme dans les collèges classiques, 1965.

France et Canada français du XVIe au XXe siècle, colloque d'histoire. Edité par Claude Galarneau et Elzéar Lavoie.

Michel Le Moignan, Le frère Antoine Bernard, historien de la Gaspésie et du peuple acadien, 1966.

André Dagenais, Révolution au Québec. Esquisse d'une république nouvelle, 1966.

Benoit Lacroix, o.p., Le Rwanda, mille heures au pays des mille collines, 1966 .

Jean-Pierre Wallot, Intrigues françaises et américaines au Canada, 18001802.

Dominique Beaudin, Le métro de Montréal, en photos et en prose, 1966.

Major crises in western civilisation, Lewis W. Spitz, Richard W. Lyman, general editor. 